\title{
Article \\ The Dependence of Ultrasonic Velocity in Ultra-Low Expansion Glass on Temperature
}

\author{
Wenqing Wei ${ }^{1,2}{ }^{\mathbb{D}}$, Yongfeng Zhang ${ }^{1,3}$, Zongzheng Du ${ }^{1}$, Minwei Song ${ }^{1}$, Yuanyuan Zhang ${ }^{1, *}$ and Hong Liu ${ }^{1,2, *}$ \\ 1 Institute of Optics and Electronics, Chinese Academy of Sciences, Chengdu 610209, China; \\ weiwenqing15@mails.ucas.ac.cn (W.W.); zhangyongfeng15@mails.ucas.edu.cn (Y.Z.); \\ 13739450124@163.com (Z.D.); 18780175291@139.com (M.S.) \\ 2 University of Chinese Academy of Sciences, Beijing 100049, China \\ 3 Key Laboratory of Adaptive Optics, Chinese Academy of Sciences, Chengdu 610209, China \\ * Correspondence: zhangyy@ioe.ac.cn (Y.Z.); liuh972@sina.com (H.L.)
}

check for updates

Citation: Wei, W.; Zhang, Y.; Du, Z.; Song, M.; Zhang, Y.; Liu, H. The Dependence of Ultrasonic Velocity in Ultra-Low Expansion Glass on Temperature. Appl. Sci. 2022, 12, 577. https://doi.org/10.3390/ app12020577

Academic Editors: Phong B. Dao, Lei Qiu, Liang Yu and Zahra Sharif Khodaei

Received: 6 December 2021 Accepted: 31 December 2021 Published: 7 January 2022

Publisher's Note: MDPI stays neutral with regard to jurisdictional claims in published maps and institutional affiliations.

Copyright: (C) 2022 by the authors. Licensee MDPI, Basel, Switzerland. This article is an open access article distributed under the terms and conditions of the Creative Commons Attribution (CC BY) license (https:/ / creativecommons.org/licenses/by/ $4.0 /)$.

\begin{abstract}
The coefficient of thermal expansion (CTE) is an important property of ultra-low expansion (ULE) glass, and the ultrasonic velocity method has shown excellent performance for the nondestructive measurement of CTE in large ULE glass. In this method, the accurate acquisition of the ultrasonic velocity in ULE glass is necessary. Herein, we present a correlation method to determine the ultrasonic TOF in ULE glass and to further obtain the ultrasonic longitudinal wave velocity $\left(c_{\mathrm{L}}\right)$ indirectly. The performance of this method was verified by simulations. Considering the dependence of $c_{\mathrm{L}}$ on temperature $(T)$, we carried out the derivation of the analytical model between $c_{\mathrm{L}}$ and $T$. Based on reasonable constant assumptions in the physical sense, a $c_{\mathrm{L}}-T$ exponential model was produced, and some experimental results support this model. Additional experiments were carried out to validate the accuracy of the $c_{\mathrm{L}}-T$ exponential model. The studies we conducted indicate that the $c_{L}-T$ exponential model can reliably predict the ultrasonic velocity in ULE glass at different temperatures, providing a means for the nondestructive CTE measurement of large ULE glass at a specified temperature.
\end{abstract}

Keywords: ultra-low expansion glass; ultrasonic velocity; correlation method; temperature coefficient; exponential model

\section{Introduction}

Ultra-low expansion (ULE) glass is a $\mathrm{SiO}_{2}-\mathrm{TiO}_{2}$ glass formed by flame hydrolysis and vapor deposition (nominal composition: $93 \mathrm{wt} \% \mathrm{SiO}_{2}$ and $7 \mathrm{wt} \% \mathrm{TiO}_{2}$ ) that has found applications in large telescope mirror blanks because of its near-zero coefficient of thermal expansion (CTE) over the $5 \sim 35^{\circ} \mathrm{C}$ temperature range $[1,2]$. However, the uniformity of glass material has a significant impact on the CTE homogeneity of the final ULE glass products, which results in figure distortion and in the degradation of the optics. It is therefore necessary to know the CTE characteristics of ULE glass to better understandand thus better control-the fabrication process for manufacturing the highest quality ULE boules.

Commonly used methods for measuring the CTE of ULE glass include the pushingrod dilatometer [3], interferometric [4,5], and photoelastic analysis [6-8] methods. All involve destructive measurements, and the procedures are time-consuming and expensive, so they are not suitable for the detection of the CTE uniformity of large ULE glass. The ultrasonic velocity in ULE glass is proportional to its CTE, a fact that can be utilized to nondestructively assess the absolute and relative CTE of large ULE glass, and its feasibility has been demonstrated by researchers $[9,10]$. In the process of using ultrasonic velocity to nondestructively detedmine CTE, there are two key points to focus on. One is how to guarantee high accuracy in the ultrasonic velocity measurements, and the second is the 
correspondence between CTE and ultrasonic velocity at certain given temperatures, mainly because both ultrasonic velocity and CTE are temperature-dependent characteristics.

For the first point, the ultrasonic velocity can be measured by various effective methods, including the threshold method [11,12], the zero-crossing method [13], the peak method [14,15], and the correlation approach [16,17]. The first three methods are based on using the local signal characteristics to measure physical quantities, and the measurement findings are strongly reliant on local signal characteristics and are easily influenced by human factors (such as subjectively selected thresholds) or noise. By contrast, the correlation method considers global signals, which contribute to its excellent noise robustness, and it is free of subjective effects. In this study, we use the correlation method to accurately measure the ultrasonic velocity in ULE glass.

We now turn to the dependence of ultrasonic velocity on temperature. The use of ultrasonic velocity measurements to characterize the CTE of ULE glass was first discovered by researchers at Corning. In their research, some troublesome operations and correction models were used to achieve nondestructive CTE testing [10]. On the one hand, the internal physical mechanism is not very clear. On the other hand, the various cumbersome practical steps (including ensuring the constant temperature of the sample to reach thermal equilibrium, removal of the water bath, quick-drying, and ultrasonic velocity measurement [9]) can introduce uncertainty regarding the sample temperature, which, in turn, affects the accuracy of the CTE measurement results. In later research, the tested sample is not forcefully separated from the constant temperature water bath during ultrasonic CTE measurement [18], which reduces the CTE measurement error, but this also increases the time cost of the entire measurement. Herein, we have analytically modeled the influencing mechanisms, which will help to simplify the practical procedures.

The paper is organized as follows. The principle and method of ultrasonic velocity measurement are mathematically stated in the following section. Section 3 summarizes the derivation of the dependence of ultrasonic velocity on temperature. Section 4 describes the composition of an ultrasonic velocity measurement system and the preparation of the tested samples in detail. In Section 5, the uncertainty and stability of the ultrasonic velocity measurement are discussed, the experimental establishment of the ultrasonic velocitytemperature exponential model is presented, and a comparison of the predicted ultrasonic velocity with the actual measured ultrasonic velocity is listed. Finally, the conclusions are presented in Section 6.

\section{Principle and Method of Ultrasonic Velocity Measurement}

\subsection{Principle of the Ultrasonic Velocity Measurement}

Many instruments are now employed to measure ultrasonic velocity, most of which use the pulse reflection method [19-21]. This method usually involves time-domain analysis based on the ultrasonic time of flight (TOF). In this analysis, the ultrasonic velocity is obtained by the ratio of the material thickness to the ultrasonic TOF [13,22]. On this basis, we built a fully integrated high-precision system to measure the ultrasonic velocity in ULE glass using the immersion pulse reflection method.

Figure 1 depicts the schematic diagram of the immersion pulse reflection method. The time of $S_{F}$ relative to the time base " 0 " point is called $t_{1}$. The time interval between $B_{1}$ and $\mathrm{S}_{\mathrm{F}}$ is recorded as $\Delta t_{1}$, and the time interval between $\mathrm{B}_{2}$ and $\mathrm{B}_{1}$ is recorded as $\Delta t_{2}$, which have the following relationship:

$$
\begin{gathered}
t_{1}=2 H / c_{\mathrm{L}}^{\text {water }} \\
\Delta t_{1}=\Delta t_{2}=2 d / c_{\mathrm{L}}^{\text {sample }}
\end{gathered}
$$




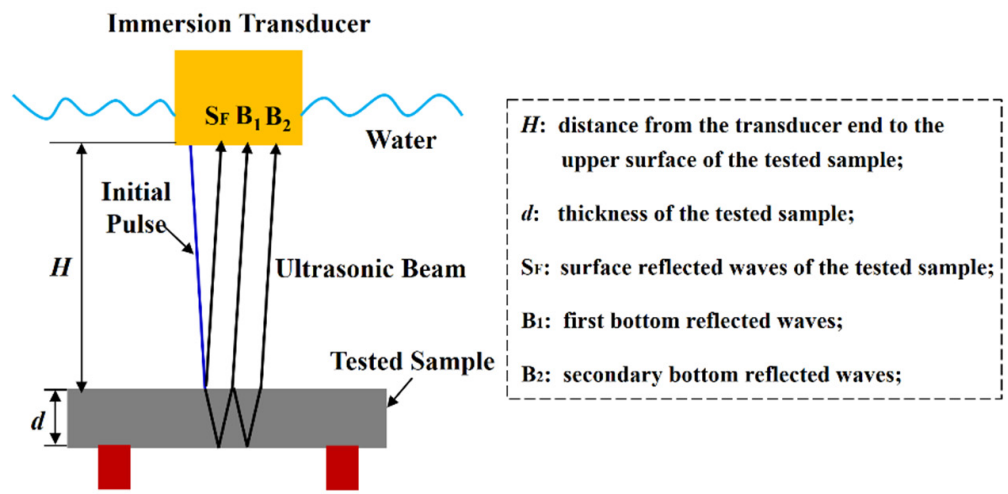

(a)

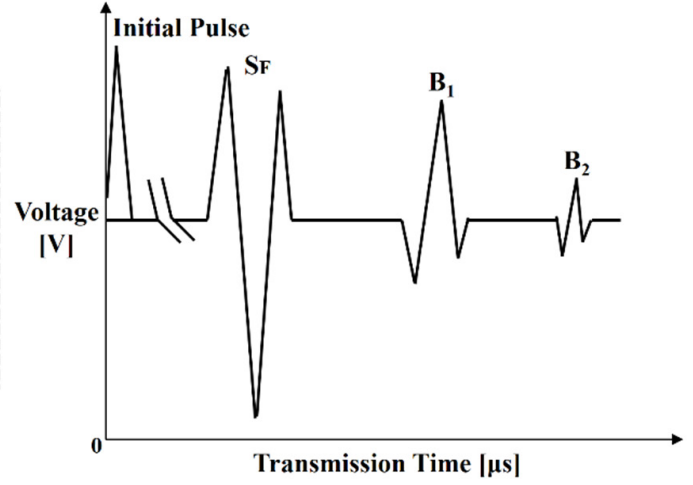

(b)

Figure 1. Schematic diagram of the immersion pulse reflection method: (a) the transmission process of ultrasonic waves; (b) the transmission time of ultrasonic waves.

The ultrasonic longitudinal wave velocity $\left(c_{\mathrm{L}}\right)$ of a sample can be calculated from Equation (1):

$$
c_{\mathrm{L}}^{\text {sample }}=2 d / \Delta t_{1}=2 d / \Delta t_{2}
$$

Using Equation (2), we can determine the $c_{\mathrm{L}}$ of the tested sample by first determining $\Delta t_{1}$ or $\Delta t_{2}$ of the ultrasonic waveform and the known thickness $d$.

\subsection{Correlation Method for Measuring the TOF}

\subsubsection{The Correlation Calculation Principle}

As the $B_{1}$ and $B_{2}$ signals of the ULE samples were readily available and were highly similar, the correlation method was used to determine the time interval between $B_{1}$ and $B_{2}$ as the ultrasonic TOF in the samples. In detail, the ultrasonic signal that was sampled and saved in the PC is designated as $x(t)$. By separating the $\mathrm{B}_{1}$ and $\mathrm{B}_{2}$ signals and noting them as $x_{1}(t)$ and $x_{2}(t)$, respectively, the correlation coefficient $(R)$ can be represented as

$$
R=\frac{\int_{-\infty}^{+\infty} x_{1}(t) x_{2}(t) \mathrm{d} t}{\sqrt{\left[\int_{-\infty}^{+\infty} x_{1}^{2}(t) \mathrm{d} t \int_{-\infty}^{+\infty} x_{2}^{2}(t) \mathrm{d} t\right]}}(|R| \leq 1) .
$$

The ultrasonic signals that were collected by the data acquisition card and the PC, on the other hand, were two discrete signal arrays. As a result, for the discrete signals, the normalized $R$ can be given as

$$
R=\frac{\sum x_{1}(i) x_{2}(i)-\sum x_{1}(i) \sum x_{2}(i) / n}{\sqrt{\left(\left[\sum x_{1}^{2}(i)-\left(\sum x_{1}(i)\right)^{2} / n\right]\left[\sum x_{2}^{2}(i)-\left(\sum x_{2}(i)\right)^{2} / n\right]\right)}}, i=1,2, \ldots, n .
$$

where $n$ is the computed length of the signal array, and $i$ is the location inside the signal array.

In the time domain of signal $x(t)$, there is a time interval between the signals $x_{1}(t)$ and $x_{2}(t)$. The time interval between $x_{1}(t)$ and $x_{2}(t)$ is the round trip time of the ultrasonic wave propagated in the thickness direction, i.e., the TOF. Equation (4) is used to generate the correlation array, with the position having the largest correlation coefficient corresponding to the temporal position of $m$. The corresponding TOF $(\Delta t)$ is equal to $m / f_{\mathrm{S}}$ if the sampling frequency is noted as $f_{\mathrm{S}}$. Finally, the following equation can be used to calculate the ultrasonic velocity:

$$
c_{\mathrm{L}}=\frac{2 d \cdot f_{\mathrm{S}}}{m} \text {. }
$$




\subsubsection{Simulation of the Correlation Method}

First, the reliability of the proposed algorithm needed to be validated by simulations in which the simulated ultrasonic signal is a declining sinusoidal function.

$$
x(t)=\beta \cdot \exp \left(-\alpha_{s} t\right) \cdot \sin \left(2 \pi f_{\mathrm{c}} t\right)
$$

where $\alpha_{\mathrm{s}}$ is the declining coefficient of the sinusoidal function set as $9 \times 10^{6} \mathrm{~Np} / \mathrm{m}$; $f_{\mathrm{c}}$ is the center frequency of the ultrasonic transducer set as $5 \mathrm{MHz} ; \beta$ is the amplitude of the signal; and the amplitudes of the initial and echo signals are set to 1 and $0.3 \mathrm{~V}$, respectively.

The sampling frequency of the signal was set as $2.5 \mathrm{GHz}$, and the TOF between the initial and echo signals was set as $17.36 \mu \mathrm{s}$. To come closer to the actual measurement signal, Gaussian noise with +15 dB SNR (Signal-to-Noise Ratio) was added to the ideal declining sinusoidal signal. The simulated signal waveform and the correlation distribution calculation results are shown in Figure $2 a, b$, respectively.

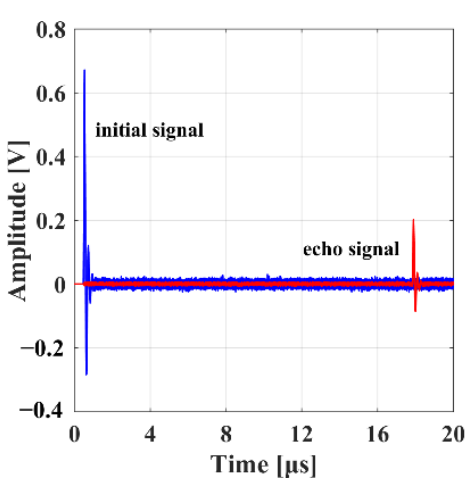

(a)

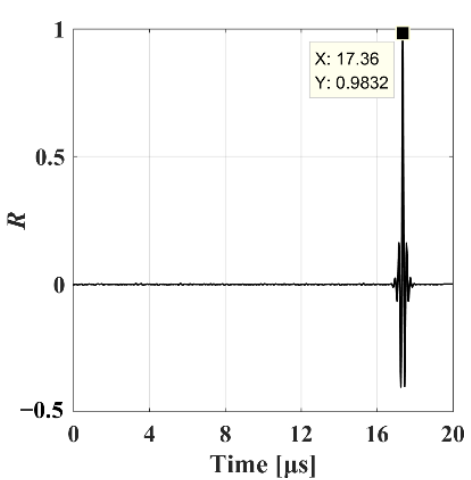

(b)

Figure 2. The correlation calculation result of a simulated signal: (a) the waveform of the simulated signal; (b) the correlation coefficient distribution.

Where the $X$ value is the time corresponding to the largest correlation coefficient, and the $\mathrm{Y}$ value is the largest correlation coefficient.

In Figure 2, the calculated correlation coefficient has a clear peak on the time axis, with a TOF of $17.36 \mu$ s corresponding to the peak. The calculated results were in good agreement with the theoretical settings, so the algorithm is suitable for ultrasonic signal processing in ultrasonic velocity measurements.

\section{Theoretical Model between Ultrasonic Velocity and Temperature}

Ultrasound is defined as an elastic wave of high frequency that propagates in a medium. Therefore, when an elastic wave propagates in an isotropic medium without being affected by volume stress, the ultrasonic longitudinal wave velocity, denoted as $c_{\mathrm{L}}$, can be found from the following equation:

$$
c_{\mathrm{L}}=\sqrt{\frac{E(1-v)}{\rho(1+v)(1-2 v)}},
$$

where $\rho$ is density, $v$ is Poisson's ratio, and $E$ is Young's modulus.

Equation (7) indicates that the $c_{\mathrm{L}}$ in the material is mainly related to Young's modulus, density, and Poisson's ratio. For ULE glass, within the upper limit of $11.5 \mathrm{wt} \%$, slight changes in the $\mathrm{TiO}_{2}$ content will not change the density or Poisson's ratio but will cause changes to Young's modulus [23]. Therefore, the difference in $c_{\mathrm{L}}$ is mainly determined by the difference in Young's modulus. Then, the effect of temperature on the ultrasonic velocity essentially reflects its effect on Young's modulus. The microscopic analysis of Young's modulus shows that the reaction of a solid to all forces comes from the potential 
energy of the interaction between atoms. The interaction potential $U(r)$ of a pair of two atoms separated by $r$ can be expressed as:

$$
U(r)=-\frac{A}{r^{n}}+\frac{B}{r^{m}}
$$

where $A, B, n$, and $m$ are all positive constants. The first term represents the energy of attraction, and the second term represents the energy of repulsion.

Assuming that the solid is stretched along the crystal axis when the tensile force changes $\mathrm{d} f$, the interatomic distance $r$ changes to $\mathrm{d} r$. At this time, the cross-sectional area $r^{2}$ of a unit cell is regarded as inconvenient. Then, Young's elastic modulus can be expressed as:

$$
E=\frac{\sigma}{\varepsilon}=\frac{\frac{\mathrm{d} f}{r^{2}}}{\frac{\mathrm{d} r}{r}}=\frac{\mathrm{d} f}{r \mathrm{~d} r},
$$

where $\sigma$ and $\varepsilon$ represent the stress and strain, respectively.

Since the binding force $f$ of the tensioned solid is only related to the first term of Equation (8), its magnitude is:

$$
f(r)=-\frac{\mathrm{d} U(r)}{\mathrm{d} r}=-\frac{n A}{r^{n+1}} .
$$

Equation (10) is then substituted into Equation (9) by deriving the derivative for $r$ to obtain

$$
E=\frac{n(n+1) A}{r^{n+3}}
$$

Assume $K=(n+1) A$ and $Q=n+3$. Equation (11) can be changed to:

$$
E=\frac{n K}{r Q} .
$$

Equation (12) takes the derivative of $T$ and divides both sides by $\operatorname{Er}^{Q}$ at the same time, shifting the term to obtain:

$$
\frac{\mathrm{d} E}{E \mathrm{~d} T}=Q \frac{\mathrm{d} r}{r \mathrm{~d} T}
$$

It is assumed that the distance between atoms still obeys the following rules when a solid is heated and expanded:

$$
r=r_{0}(1+\alpha T),
$$

where $r_{0}$ is the atomic distance when the absolute temperature $T_{0}=0 ; \alpha$ is the linear expansion coefficient of the solid, and its differential definition is:

$$
\left\{\alpha=\frac{1}{r} \frac{d r}{d T}, \eta=\frac{1}{E} \frac{d E}{d T} .\right.
$$

The $\eta$ in Equation (15) is the temperature coefficient of the elastic modulus. Relevant studies have demonstrated that the elastic modulus of ULE glass increases with the increase in temperature, and the increment $\mathrm{d} E$ of $E$ has a positive value [23]. When both sides of Equation (13) are multiplied by dT, Equation (14) is then substituted in and integrated, and we obtain:

$$
\int_{E_{0}}^{E} \frac{\mathrm{d} E}{E}=Q \int_{T_{0}}^{T} \frac{\mathrm{d}(1+\alpha T)}{(1+\alpha T)} .
$$

From Equation (16) and considering $T_{0}=0$, we obtain:

$$
E=E_{0}\left(\frac{1+\alpha T}{1+\alpha T_{0}}\right)^{Q} \approx E_{0}(1+Q \alpha T)
$$


From Equations (13) and (15), we see that:

$$
Q=\eta / \alpha .
$$

Therefore, Equation (17) is changed to:

$$
E=E_{0}(1+\eta T)
$$

Equation (19) shows that the elastic modulus of ULE glass increases as the temperature increases, where $\eta$ is the temperature coefficient of the elastic modulus. Therefore, it can be inferred that the $c_{\mathrm{L}}$ in ULE glass also increases as $T$ increases. A review of the data reveals that when considering the effects of thermal expansion, there is a specific equation relating the $c_{\mathrm{L}}$ and $T$, which is as follows [24]:

$$
c_{\mathrm{L}}^{T}=c_{\mathrm{L}}^{T_{0}}\left[1+\alpha_{\mathrm{L}}\left(T-T_{0}\right)\right]
$$

where $\alpha_{\mathrm{L}}$ is the temperature coefficient of the ultrasonic longitudinal wave velocity, a positive value of about $10^{-4}$ orders of magnitude, and $c_{\mathrm{L}}^{T}, c_{\mathrm{L}}^{T_{0}}$ indicate the ultrasonic longitudinal wave velocity of the material at temperatures $T$ and $T_{0}$, respectively.

Although there are certain differences in $\alpha_{\mathrm{L}}$ at different temperature points, we can use the $c_{\mathrm{L}}-T$ data in a small temperature range to fit and solve the average $\alpha_{\mathrm{L}}$ in this temperature range. The method of a differential equation is introduced here to obtain the mathematical relationship between $c_{\mathrm{L}}$ and $T$ in a small temperature range. Writing Equation (20) in a differential form, we obtain

$$
\alpha_{\mathrm{L}}=\frac{\mathrm{d} c_{\mathrm{L}}}{\mathrm{c}_{\mathrm{L}} \mathrm{d} T}, T_{1}<T<T_{2}
$$

Solving this equation yields

$$
c_{\mathrm{L}}=c^{\prime} \cdot e^{\alpha_{\mathrm{L}} T}
$$

Equation (22) shows that the relationship between $c_{\mathrm{L}}$ and $T$ is theoretically exponential within a certain temperature range.

\section{Tested Material and Experimental Setup}

\subsection{Materials and Sample Preparation}

The selected ULE glass was Corning Code 7972 glass. Considering the boundary effect of ultrasonic wave propagation, the experimental samples were prepared in a cylindrical shape with a cross-sectional area that was larger than that of the ultrasonic transducer. To avoid the adverse effects of the scattering attenuation of ultrasound at the interface of the sample, the two end faces of the tested sample should be flat and parallel to each other. As shown in Figure 3, six cylindrical glass samples were cut with an equal thickness (50-0.1 mm) along the radial position of the ULE 7972 boule (No. 82714) using an abrasive water jet; then, these samples were finely ground and polished to achieve a flatness of $0.5 \lambda$ and parallelism of $20 \mu \mathrm{m}$. The six samples were numbered $1 \# \sim 6 \#$ from the inside to the outside of the boule according to the increasing CTE. The details of the ULE samples are summarized in Table 1.

\subsection{Ultrasonic Measurement System}

The schematic diagram of the $c_{\mathrm{L}}$. measurement experimental system is illustrated in Figure 4. A $75 \mathrm{MHz}$ ultrasonic pulser/receiver (Olympus, Waltham, MA, USA, model 5073PR) was used, which was connected to a $400 \mathrm{MHz}$ data acquisition card (Spectrum Instrumentation $\mathrm{GmbH}$, Grosshansdorf, Germany, model M4i.2220-x8) that transmits the ultrasonic signals to a computer to be processed. All signals were captured with $2,500,000$ points at a sampling rate of $2.5 \mathrm{GHz}$. After the acquisition, the data were properly processed to determine the involved ultrasonic velocities. The temperature of the tested 
sample was controlled by a thermostatic water tank (Hangzhou Qiwei, Hangzhou, China, model DHC-05-B) with a temperature control precision of $\pm 0.05^{\circ} \mathrm{C}$.

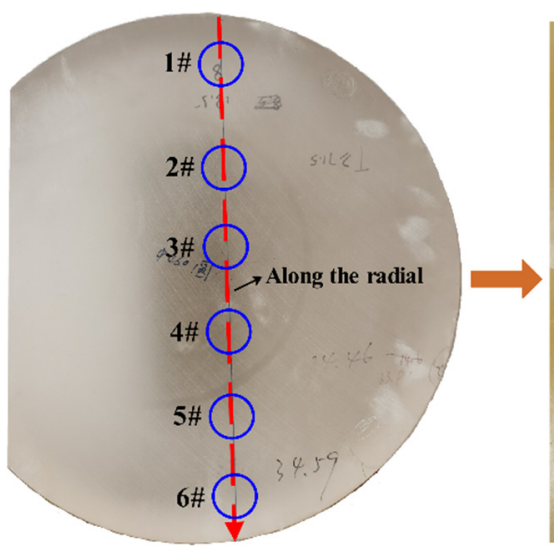

(a)

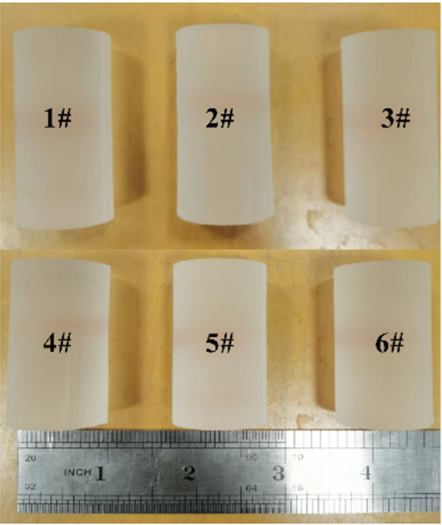

(b)

Figure 3. The prepared ULE glass samples: (a) source of samples; (b) photograph of samples.

Table 1. The important parameters of the ULE samples.

\begin{tabular}{ccc}
\hline No. & $\begin{array}{c}\text { Thickness } \\
(\mathbf{m m})\end{array}$ & $\begin{array}{c}\mathrm{CTE}\left(5 \sim 35^{\circ} \mathrm{C}\right) \\
\left(\mathbf{p p b} /{ }^{\circ} \mathrm{C}\right)\end{array}$ \\
\hline 1\# & 49.936 & -1 \\
2\# & 49.932 & 0 \\
3\# & 49.937 & 1 \\
$4 \#$ & 49.940 & 2 \\
$5 \#$ & 49.941 & 2 \\
6\# & 49.927 & 3
\end{tabular}

Note: No.-sample number; thickness-measured thickness of the ULE samples; CTE $\left(5 \sim 35^{\circ} \mathrm{C}\right)$-average CTE over the temperature range $5 \sim 35^{\circ} \mathrm{C} ; \mathrm{ppb} /{ }^{\circ} \mathrm{C}$ - unit of $\mathrm{CTE}\left(1 \mathrm{ppb} /{ }^{\circ} \mathrm{C}=1 \times 10^{-9} /{ }^{\circ} \mathrm{C}\right)$.

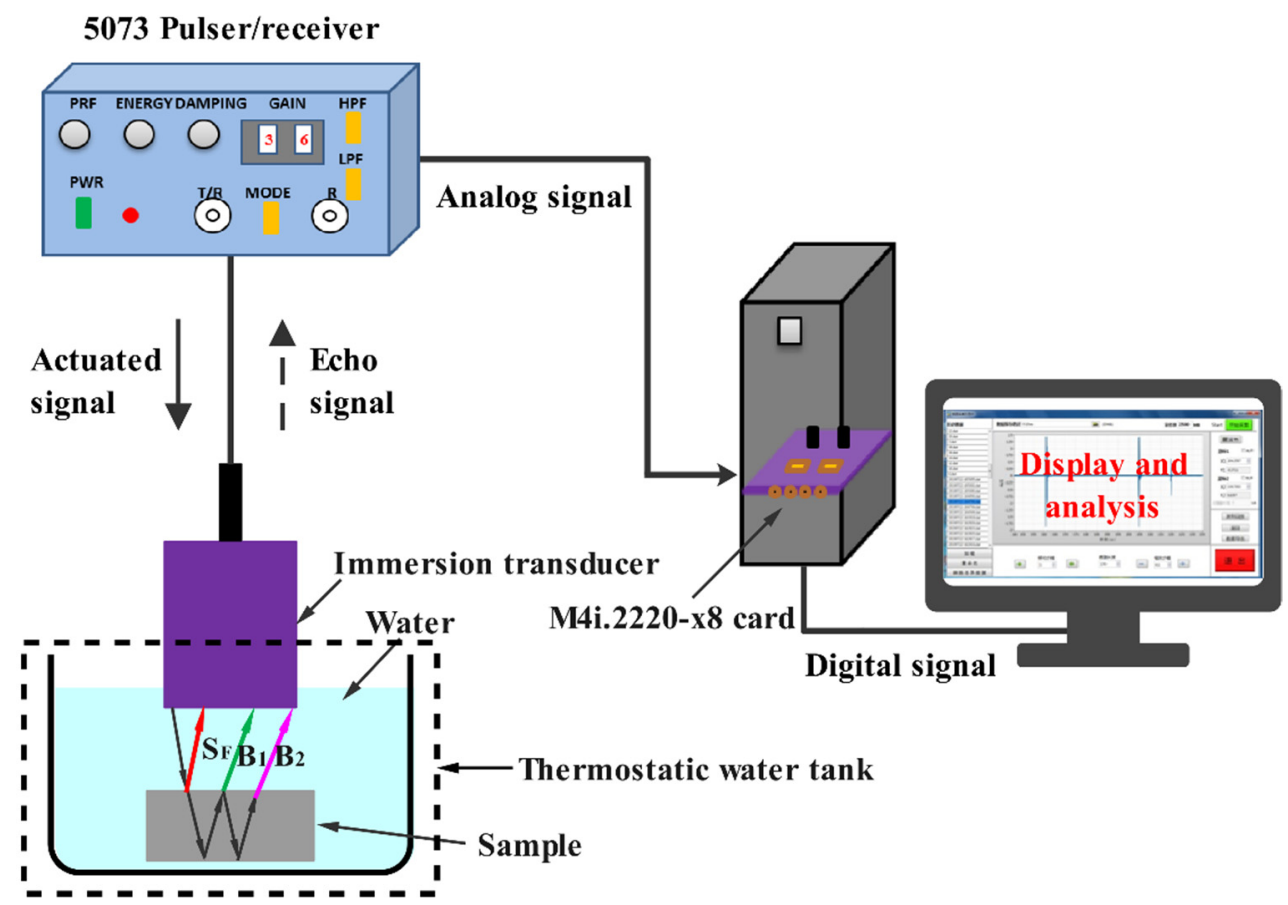

Figure 4. Schematic diagram of the experimental system for measuring ultrasonic velocity. 
The self-generating and self-receiving transducer model was utilized to transmit and receive the ultrasonic waves. Considering the diameter and thickness of the tested sample, wideband focusing $19.05 \mathrm{~mm}$ diameter longitudinal wave immersion transducers (Olympus, Waltham MA, USA) with 3.5 and $5 \mathrm{MHz}$ nominal center frequencies were employed in this investigation. Figure 5 illustrates the ultrasonic velocity measurement results for the samples $1 \# \sim 6 \#$ at $20^{\circ} \mathrm{C}$ using two different ultrasonic frequencies: 3.5 and $5 \mathrm{MHz}$. Each sample was subjected to five replicate measurements by each transducer. The ultrasonic frequency had little effect on the ultrasonic velocity of the sample, as the $c_{\mathrm{L}}$ in the sample only depends on its inherent physical parameters, including its bulk modulus and density [25]. In addition, the repeatability of the $c_{\mathrm{L}}$ measurement was essentially the same for both frequency transducers, both within $0.1 \mathrm{~m} / \mathrm{s}$. This suggests that any frequency could be used to characterize the CTE of ULE glass in engineering applications when using ultrasonic velocity measurement methods.

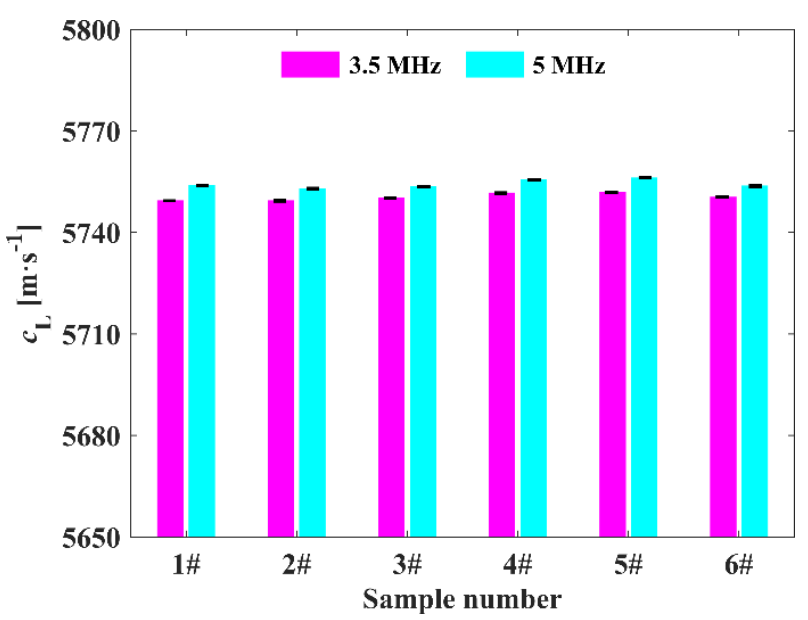

Figure 5. $c_{\mathrm{L}}$ measurement results of the ULE samples 1\# 6\# at frequencies of 3.5 and $5 \mathrm{MHz}$.

\section{Results and Discussion}

\section{1. $c_{L}$ Measurement at a Single Temperature}

\subsubsection{Uncertainty Analysis}

In this work, an ultrasonic transducer with a $5 \mathrm{MHz}$ center frequency was employed to measure the ultrasonic velocities in all of the prepared ULE samples. Figure 6 gives an example of the received ultrasonic signals, which formed a series of ultrasonic longitudinal waves with a sampling rate of $2.5 \mathrm{GHz}$. High-frequency noise was found to be present in the actual ultrasonic signals. However, since the correlation of the noisy signal was very small, the effect of such noise was removed via the correlation calculation. The results of the TOF calculations obtained using this method are depicted in Figure 6. Again, there is a peak in the correlation coefficient distribution plot corresponding to a TOF of $\Delta t=17.3383 \mu$ s.

The uncertainty in measuring ultrasonic velocity was also investigated, and the expression is shown in Equation (23).

$$
u_{c_{\mathrm{L}}}=\sqrt{\left(\frac{2}{\Delta t} u_{d}\right)^{2}+\left(\frac{2 d}{(\Delta t)^{2}} u_{\Delta t}\right)^{2}}
$$

where $u_{c_{L}}, u_{d}$, and $u_{\Delta t}$ are the uncertainties regarding the $c_{L}, d$, and TOF in the ULE glass, respectively.

As illustrated in the first item of Equation (23), the uncertainty related to the $d$ was $u_{c_{\mathrm{L}}}(d)=(2 / \Delta t) \Delta u_{d}$. A typical value of $\Delta t$ is $17.3562 \mu \mathrm{s}$. When measuring the $d$ of the ULE sample, $u_{d}$ was evaluated by the measurement precision of a micrometer $(0.001 \mathrm{~mm})$, thus $u_{c_{\mathrm{L}}}(d)=0.12 \mathrm{~m} / \mathrm{s}$. After substituting the measured values into the second item of Equation (23), the uncertainty introduced by the TOF $(\Delta t)$ was obtained: $u_{c_{\mathrm{L}}}(\Delta t)=0.332 \times 10^{9} u_{\Delta t}$. 
Since $u_{\Delta t}$ was evaluated by the sampling period of the data acquisition card ( $\left.0.4 \mathrm{~ns}\right)$, the typical $u_{c_{\mathrm{L}}}(\Delta t)$ was $0.13 \mathrm{~m} / \mathrm{s}$. Therefore, when measuring the ULE glass using the proposed experimental setup, the calculated total uncertainty was $u_{c_{\mathrm{L}}}=0.2 \mathrm{~m} / \mathrm{s}$, ensuring that the ultrasonic velocity measurements at a single temperature had high reliability.

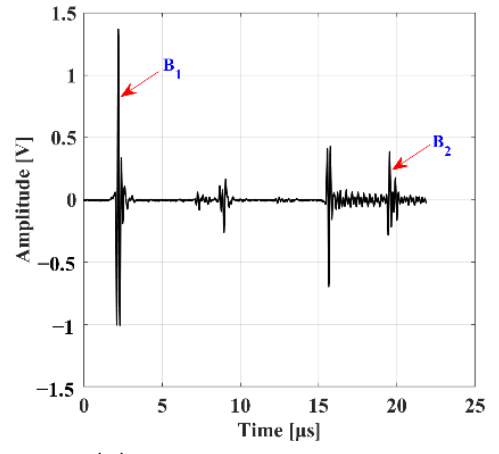

(a)

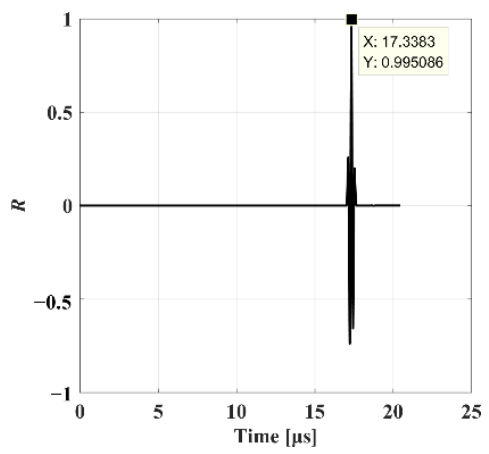

(b)

Figure 6. The correlation calculation result of an actual received ultrasonic signal: (a) the ultrasonic echo signal; (b) the correlation coefficient distribution.

\subsubsection{Stability of $c_{\mathrm{L}}$ Measurement}

To determine whether the proposed measurement system could provide reliable $c_{\mathrm{L}}$ measurements over long periods of time, the stability of the measured $c_{\mathrm{L}}$ value also needs to be considered when the tested samples reach thermal equilibrium. Long-term measurement of the ultrasonic velocity in samples 1\# 6\# with a high CTE was taken at the same temperature $\left(20^{\circ} \mathrm{C}\right)$, and the $c_{\mathrm{L}}$ data were recorded at $1 \mathrm{~h}$ intervals, for a total of seven measurements in one day. Figure 7 depicts the variation in $c_{\mathrm{L}}$ with the measurement time.

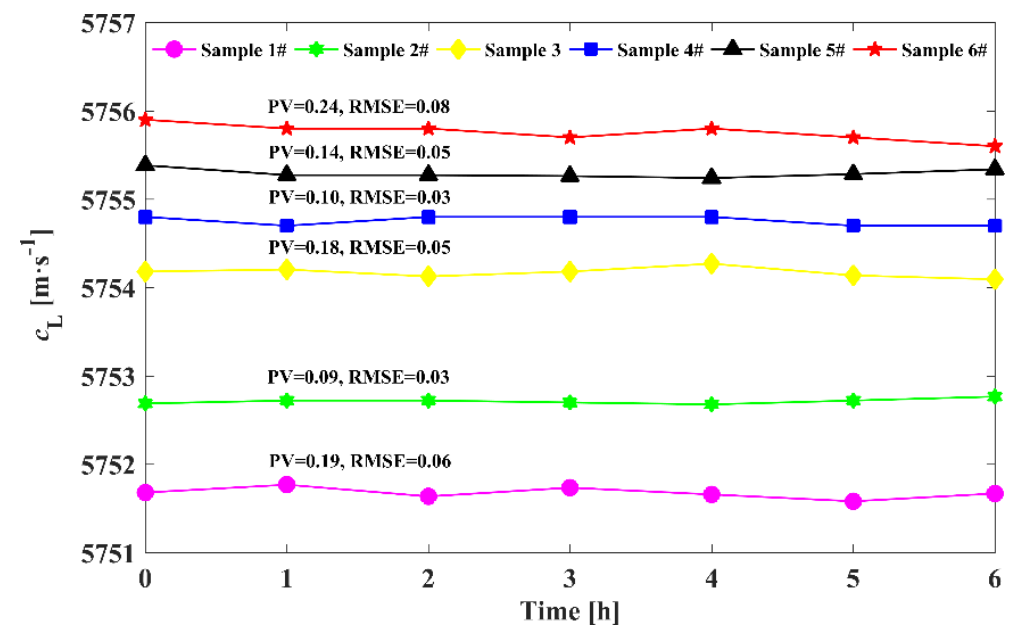

Figure 7. The change in $c_{\mathrm{L}}$ with time for samples $1 \# \sim 6 \#$.

The $c_{\mathrm{L}}$ measured in the same sample was almost constant at the different times while maintaining a constant temperature, and the RMSE (Root Mean Squared Error) of the $c_{\mathrm{L}}$ changes were all within $0.10 \mathrm{~m} / \mathrm{s}$, which indicate that the measurement system that was built in this paper has stable performance. This also provides a strong guarantee for the $c_{\mathrm{L}}$ measurement of a large batch of ULE glass samples.

\subsection{Measurement and Analysis of $c_{L}-T$ Data}

\subsubsection{Acquisition of $c_{\mathrm{L}}-\mathrm{T}$ Data}

The tested sample and an ultrasonic transducer fixed above the sample were placed into a thermostatic water tank, and the temperature in the tank was steadily increased 
in the range of $10 \sim 30{ }^{\circ} \mathrm{C}$. Here, the temperature range of $10 \sim 30{ }^{\circ} \mathrm{C}$ was chosen for two reasons: (1) this corresponds to the real temperature range that ULE glass is likely to experience when undergoing ultrasonic measurements, i.e., the range of room temperature throughout the year, and (2) it corresponds to the temperature range of the ultrasonic transducer in use. We were utilizing a standard immersion transducer, whose normal operating temperature range is $10 \sim 60{ }^{\circ} \mathrm{C}$, beyond which the piezoelectric action of the transducer would be weakened, making high-amplitude data acquisition difficult. The ultrasonic echo signals were manually sampled and stored by the PC at a temperature interval of $1{ }^{\circ} \mathrm{C}$, which was chosen to account for the maximum number of temperature points to be sampled and the required modeling time. The temperature was held constant within $\pm 0.05^{\circ} \mathrm{C}$, as measured using a digital thermometer probe in the bath.

To ensure that the glass sample was in thermal equilibrium during the TOF measurement, the sample was immersed in a controlled water bath for at least $94 \mathrm{~min}$, which was calculated according to the time $t$ (hours) to reach equilibrium for a given glass thickness $d(\mathrm{~cm})$, which could be expressed as $t=d^{2} / 16$ in [26]. To reduce measurement errors, the echo signals were acquired three times at each temperature point. By using the described correlation algorithm method, the ultrasonic TOF was obtained, and the average values were used to calculate the $c_{\mathrm{L}}$ of the tested ULE samples. Nevertheless, the experiment did not consider the change in the $d$ and $\rho$ of the tested samples.

The obtained ultrasonic velocities of samples 1\# 6\# are presented in Figure 8 in terms of temperature. The ultrasonic velocities are observed to increase as the temperature increases. Changes in $c_{\mathrm{L}}$ are correlated with temperature changes, regardless of the absolute value of the CTE. Comparing Figure $8 \mathrm{a}-\mathrm{f}$, we can also observe that the ultrasonic velocity differs at the same temperature and that the $c_{\mathrm{L}}$ increases with the CTE of ULE glass.

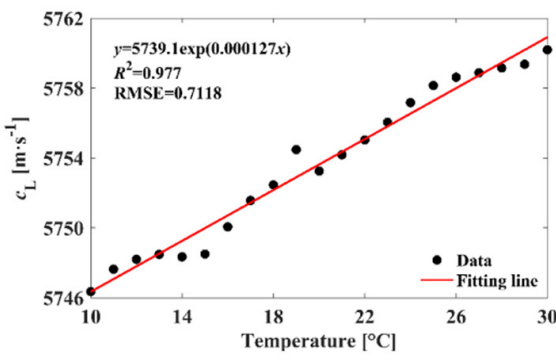

(a)

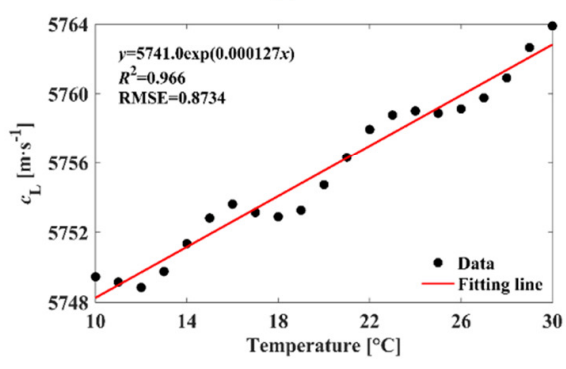

(d)

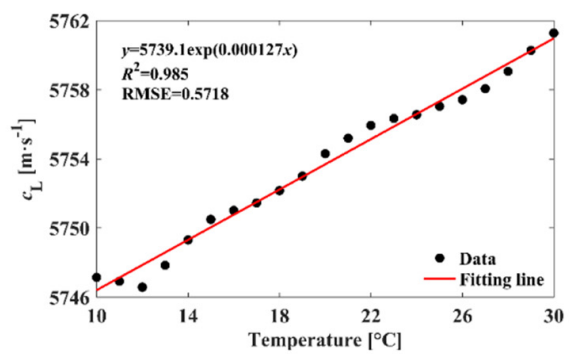

(b)

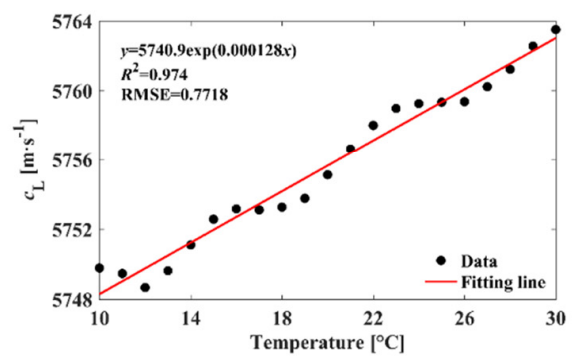

(e)

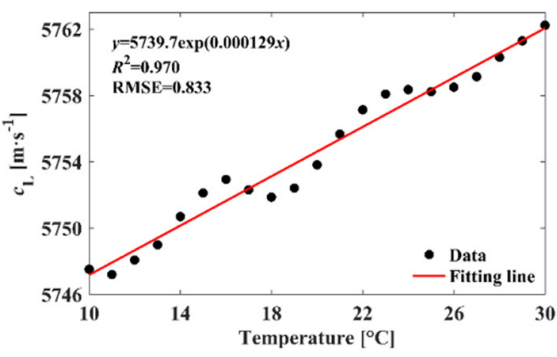

(c)

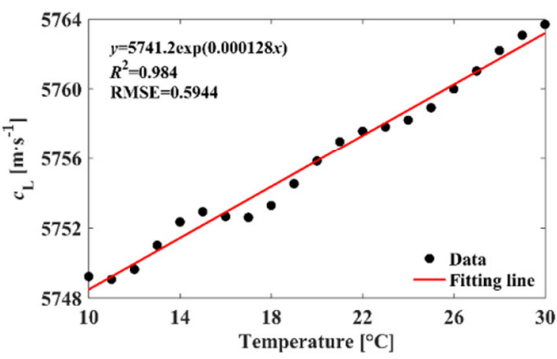

(f)

Figure 8. Changes in $c_{\mathrm{L}}\left(\mathrm{m} \cdot \mathrm{s}^{-1}\right)$ with temperature for all measured samples: (a) sample 1\#; (b) sample 2\#; (c) sample 3\#; (d) sample 4\#; (e) sample 5\#; (f) sample 6\#.

\subsubsection{Analysis of the Change in the $c_{\mathrm{L}}$ with $T$}

The data from Figure 8 were used to directly plot the changes in $c_{\mathrm{L}}$ as a function of $T$ according to the exponential fitting in Equation (22). Table 2 displays the results of all six samples, with excellent exponential fits given for each case and with $\alpha_{\mathrm{L}}$ mostly ranging from 0.000127 to 0.000129 , which shows that for ULE materials with very small CTE differences, their $\alpha_{\mathrm{L}}$ is almost constant. This means that the temperature effect pattern on ultrasonic velocity was essentially the same in ULE glasses with different CTE values. 
Therefore, the average value of these $\alpha_{\mathrm{L}}$ coefficients could be taken as the temperature correction coefficient of ultrasonic velocity in ULE glass. Of course, because of the small difference in the CTE, the $c^{\prime}$ in Equation (22) showed relatively large fluctuations. In the physical sense, the $c^{\prime}$ indicates that $c_{\mathrm{L}}$ correpsonds to $0{ }^{\circ} \mathrm{C}$. It was found that as the CTE of the ULE sample increased, $c^{\prime}$ increased correspondingly, which is consistent with the mechanism of the linear positive correlation between $c_{\mathrm{L}}$ and the CTE.

Table 2. Data from the six samples shown in Figure 8 were taken to exponentially fit the relationship between $c_{\mathrm{L}}$ and $T$.

\begin{tabular}{cccc}
\hline No. & $\boldsymbol{\alpha}_{\mathbf{L}} / \times \mathbf{1 0}^{-\mathbf{6}}$ & $\boldsymbol{R}^{\mathbf{2}}$ & RMSE \\
\hline $1 \#$ & 127 & 0.977 & 0.7118 \\
$2 \#$ & 127 & 0.985 & 0.5718 \\
$3 \#$ & 129 & 0.970 & 0.8330 \\
$4 \#$ & 127 & 0.966 & 0.8734 \\
$5 \#$ & 128 & 0.974 & 0.7718 \\
$6 \#$ & 128 & 0.984 & 0.5944 \\
\hline
\end{tabular}

Note: No.-sample number; $\alpha_{\mathrm{L}}$ 一temperature coefficient of ultrasonic longitudinal wave velocity; $R^{2}-R$-square (a measure of goodness of fit).

To represent the more pronounced difference in the ultrasonic velocity with increasing temperature for the ULE samples with different CTE values, a new coefficient is defined as follows:

$$
\beta_{\mathrm{L}}=\frac{\Delta c_{\mathrm{L}}}{c_{\mathrm{L}} \Delta T}
$$

Here, we take the $c_{\mathrm{L}}$ corresponding to $10{ }^{\circ} \mathrm{C}$ of the samples as the basis $c_{\mathrm{L}}$, and calculate the $\beta_{\mathrm{L}}$ via Equation (24) with temperature increments of $5^{\circ} \mathrm{C}$, i.e., at $15^{\circ} \mathrm{C}, 20^{\circ} \mathrm{C}$, $25^{\circ} \mathrm{C}$, and $30^{\circ} \mathrm{C}$. The $\beta_{\mathrm{L}}$ for samples $1 \#, 2 \#$, and $3 \#$ is shown in Figure 9 . We noted that for the different temperature ranges, the speed of the ultrasonic velocity changes with the temperature was inconsistent. This is possibly because the elastic modulus also has a temperature coefficient $\beta_{E}$, which varies in different temperature ranges. The PV value of $\beta_{\mathrm{L}}$ for the three samples was $0.000098,0.000056$, and 0.000101 . Considering that the difference was minor, we conclude that the multi-data point fitting method that was used to obtain $\alpha_{\mathrm{L}}$ in a given temperature range in this paper is a more feasible equivalent to an average function and has wider temperature applicability.

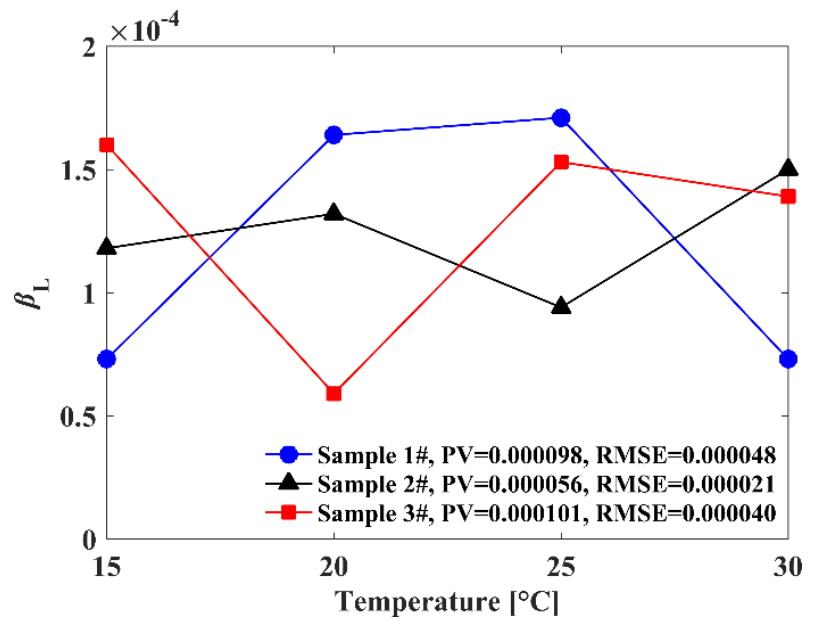

Figure 9. The relationship between $\beta_{\mathrm{L}}$ and temperature for ULE samples with different CTEs.

Samples 4\# and 5\#, which had the same CTE, were also analyzed for differences in the $c_{\mathrm{L}}$ as the temperature increased, and the results are shown in Figure 10. We found that the two samples had similar patterns of variation in $\beta_{\mathrm{L}}$. This implies that the changing trends in the ultrasonic velocity with temperature was, to some extent, consistent with the 
changing trend of the CTE with temperature; however, this assumes that the thickness of the ULE glass sample did not change over a wide temperature range.

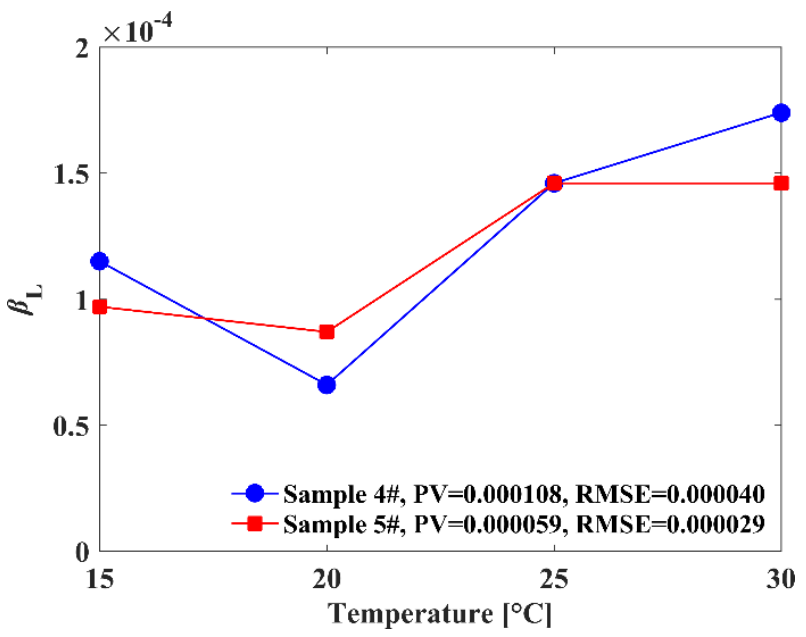

Figure 10. The relationship between $\beta_{\mathrm{L}}$ and temperature for ULE samples with the same CTE.

The correlation between $c_{\mathrm{L}}$ and $T$ in ULE glass over a wide range of CTE values from $-1 \mathrm{ppb} /{ }^{\circ} \mathrm{C}$ to $+3 \mathrm{ppb} /{ }^{\circ} \mathrm{C}$ was investigated and discussed. We will next validate the accuracy of the $c_{L}-T$ exponential model for ULE samples with different CTE values through a series of experiments.

\subsection{Accuracy Validation of $c_{L}-T$ Model}

The accuracy of the $c_{\mathrm{L}}-T$ model was decisive for its application in engineering. Samples 1\#, 3\#, and 5\# were randomly selected, and their ultrasonic velocities were measured at ten random temperature points in the range of $10 \sim 30^{\circ} \mathrm{C}$. The measured values were compared with the model predictions in this paper, and the results are shown in Table 3.

Table 3. Experimental data from the model validation and their error analysis.

\begin{tabular}{cccccc}
\hline No. & $\begin{array}{c}\text { Temperature } \\
\left({ }^{\circ} \mathbf{C}\right)\end{array}$ & $\boldsymbol{c}_{\mathbf{m}}(\mathbf{m} / \mathbf{s})$ & $\boldsymbol{c}_{\mathbf{p}}(\mathbf{m} / \mathbf{s})$ & $\boldsymbol{c}_{\mathbf{m}}-\boldsymbol{c}_{\mathbf{p}}(\mathbf{m} / \mathbf{s})$ & $\begin{array}{c}|\delta| \\
\%\end{array}$ \\
\hline & 10.2 & 5745.5 & 5746.3 & -0.8 & 0.014 \\
& 12.7 & 5748.1 & 5748.2 & -0.1 & 0.002 \\
& 14.3 & 5749.6 & 5749.3 & 0.3 & 0.005 \\
& 15.2 & 5749.4 & 5750.0 & -0.6 & 0.010 \\
$1 \#$ & 17.6 & 5750.3 & 5751.7 & -1.4 & 0.024 \\
& 21.8 & 5754.4 & 5754.8 & -0.4 & 0.007 \\
& 23.5 & 5754.9 & 5756.1 & -1.2 & 0.021 \\
& 25.6 & 5457.1 & 5757.6 & -0.5 & 0.009 \\
& 26.0 & 5757.5 & 5757.9 & -0.4 & 0.007 \\
& 27.9 & 5759.4 & 5759.3 & 0.1 & 0.002 \\
& 10.8 & 5747.5 & 5746.8 & $\sigma_{c}=0.75$ & 0.012 \\
& 12.4 & 5747.9 & 5748.0 & -0.1 & 0.002 \\
& 15.2 & 5751.7 & 5750.1 & 1.6 & 0.028 \\
& 16.5 & 5751.5 & 5751.0 & 0.5 & 0.009 \\
& 16.9 & 5751.4 & 5751.3 & 0.1 & 0.002 \\
& 18.4 & 5751.5 & 5752.4 & -0.9 & 0.016 \\
& 23.3 & 5757.3 & 5756.1 & 1.2 & 0.021 \\
& 24.3 & 5757.2 & 5756.8 & 0.4 & 0.007 \\
& 25.6 & 5757.5 & 5757.8 & -0.3 & 0.005 \\
& 29.0 & 5761.1 & 5760.3 & 0.8 & 0.014 \\
& & & $\sigma_{c}=0.85$ & \\
\hline
\end{tabular}


Table 3. Cont.

\begin{tabular}{cccccc}
\hline No. & $\begin{array}{c}\text { Temperature } \\
\left({ }^{\circ} \mathbf{C}\right)\end{array}$ & $\boldsymbol{c}_{\mathbf{m}}(\mathbf{m} / \mathbf{s})$ & $c_{\mathbf{p}}(\mathbf{m} / \mathbf{s})$ & $c_{\mathbf{m}}-c_{\mathbf{p}}(\mathbf{m} / \mathbf{s})$ & $\begin{array}{c}|\delta| \\
\%\end{array}$ \\
\hline 10.6 & 5750.4 & 5749.7 & 0.7 & 0.012 \\
& 11.3 & 5750.2 & 5750.2 & 0.0 & 0.000 \\
$5 \#$ & 13.5 & 5752.1 & 5751.8 & 0.3 & 0.005 \\
& 14.1 & 5753.1 & 5752.3 & 0.8 & 0.014 \\
& 15.1 & 5754.4 & 5753.0 & 1.4 & 0.024 \\
& 17.5 & 5754.2 & 5754.8 & -0.6 & 0.010 \\
& 20.3 & 5756.8 & 5756.8 & 0.0 & 0.000 \\
& 25.2 & 5760.3 & 5760.5 & -0.2 & 0.003 \\
& 25.9 & 5760.4 & 5761.0 & -0.6 & 0.010 \\
& 29.9 & 5764.8 & 5763.9 & 0.9 & 0.016 \\
\hline
\end{tabular}

Note: $c_{\mathrm{m}}$ is the actual measured ultrasonic velocity, $c_{\mathrm{p}}$ is the ultrasonic velocity predicted by the fitting model, and $\delta$ denotes the relative error. The deviation $\sigma_{c}$ is a measure of the inaccuracy.

Considering that the measurement locations of the three samples in the model validation procedure may vary, it was reasonable to use a recalculation of $c^{\prime}$ based on the standard ultrasonic velocity at $20^{\circ} \mathrm{C}$ to determine the $c_{\mathrm{L}}-T$ exponential model, which may differ slightly from the $c^{\prime}$ obtained by modeling the above $c_{\mathrm{L}}-T$ data. The $c_{\mathrm{L}}$ values in the tested glasses at an average room temperature of $20^{\circ} \mathrm{C}$ were substituted into the derived exponential model based on the average temperature coefficient from the above fitting analysis to determine a $c_{\mathrm{L}}-T$ exponential model for each tested glass. As seen in Table 3 , the standard deviation of the predicted values for three samples were all within $0.90 \mathrm{~m} / \mathrm{s}$, and the relative errors between measured and model-predicted values were mostly within $0.020 \%$, which suggests that the models fitted in this work exhibit high precision.

Of course, it should be noted that increasing the sample thickness may further reduce the errors in $c_{\mathrm{L}}$ measurement, but it also imposes more stringent requirements on the frequency of the transducer and time required for the sample to reach thermal equilibrium.

\section{Conclusions}

In this investigation, the aim was to theoretically analyze and validate the dependence of the ultrasonic velocity in ULE glass on temperature. Based on the simulation and experimental investigations, we can draw the following conclusions:

1. The proposed pulse reflection immersion method provides reliable and stable measurements of the ultrasonic echo signal of ULE glass, and the calculation of the signal based on the correlation method can be used to conveniently and accurately extract the ultrasonic TOF of the tested sample and then obtain the ultrasonic velocity.

2. The application of the proposed method for six ULE samples with different CTE values indicates that ultrasonic velocity increases as the experimental temperature is increased. Furthermore, the $c_{\mathrm{L}}-T$ exponential model was theoretically analyzed and experimentally fitted. The predicted $c_{\mathrm{L}}$ using the exponential model at ten random temperature points shows good agreement with the actual measured ultrasonic velocities at the same temperature.

These findings indicate the promising potential of the $c_{\mathrm{L}}-T$ exponential model to determine the ultrasonic velocity at a specified temperature for ultrasonic nondestructive CTE measurement in large ULE glass, which will be critical for reliably evaluating the CTE homogeneity of large ULE glass at a specified temperature.

There are some comments that can be made about the general applicability of our method. In this paper, the derivation and verification of the $c_{L}-T$ relationship are only for isotropic ULE glass, but the method for deriving exponential model can also be applied to anisotropic materials, such as anisotropic crystals, and for obtaining the longitudinal wave velocity, transverse wave velocity, and then the Poisson's ratio. The only distinction is $\alpha_{\mathrm{L}}$. As for the experimental verification method for the exponential model, the correlation 
method, it is also applicable for the measurement of the TOF, regardless of whether the material is isotropic.

Author Contributions: Conceptualization, W.W., Y.Z. (Yuanyuan Zhang) and H.L.; methodology, Y.Z (Yongfeng Zhang) and H.L.; software, W.W. and Y.Z. (Yongfeng Zhang); validation, W.W. and H.L.; investigation, W.W. and M.S.; resources, Y.Z. (Yuanyuan Zhang), M.S. and H.L.; data curation, W.W.; writing-original draft preparation, W.W.; writing-review and editing, Y.Z. (Yongfeng Zhang), Z.D. and H.L.; visualization, W.W.; project administration, Y.Z. (Yuanyuan Zhang). All authors have read and agreed to the published version of the manuscript.

Funding: This research received no external funding.

Institutional Review Board Statement: Not applicable.

Informed Consent Statement: Not applicable.

Data Availability Statement: Not applicable.

Conflicts of Interest: The authors declare no conflict of interest.

\section{References}

1. VanBrocklin, R.R.; Edwards, M.J.; Wells, B. Review of Corning's capabilities for ULE mirror blank manufacturing for an extremely large telescope. In Proceedings of the SPIE-The International Society for Optical Engineering, Orlando, FL, USA, 6 July 2006; Volume 6273, p. 627301.

2. Kendrick, S.E.; Stahl, H.P. Large aperture space telescope mirror fabrication trades. In Proceedings of the SPIE-The International Society for Optical Engineering, Marseille, France, 12 July 2008; Volume 7010, p. 70102G.

3. Plummer, W.A.; Hagy, H.E. Precision thermal expansion measurements on low expansion optical materials. Appl. Opt. 1968, 7, 825-831. [CrossRef]

4. Imai, H.; Okaji, M.; Kishii, T.; Sagara, H.; Aikawa, H.; Kato, R. Measurement of thermal expansivity of low-expansion glasses by interferometric methods: Results of an interlaboratory comparison. Int. J. Thermophys. 1990, 11, 937-947. [CrossRef]

5. Kato, R.; Azumi, T.; Maesono, A. Measurement of thermal expansion of low-expansion glasses by a laser interferometric thermal expansion meter. High Temp.-High Press. 1991, 23, 615-620.

6. Hagy, H.E.; Smith, A.F. Sandwich seal in the development and control of sealing glasses. J. Can. Ceram. Soc. 1969, 38, $63-68$.

7. Hagy, H.E.; Best, M.E. Comparison of two high-precision nondestructive measurement methods for evaluating thermal expansion differences in the 8.3-m ultralow-expansion Subaru primary mirror blank. Appl. Opt. 1996, 35, 1126-1128. [CrossRef]

8. Gulati, S.T.; Hagy, H.E. Theory of the narrow sandwich seal. J. Am. Ceram. Soc. 2006, 61, 260-263. [CrossRef]

9. Hagy, H.E. High precision photoelastic and ultrasonic techniques for determining absolute and differential thermal expansion of titania-silica glasses. Appl. Opt. 1973, 12, 1440-1446. [CrossRef] [PubMed]

10. Hagy, H.E.; Shirkey, W. Determining absolute thermal expansion of titania-silica glasses: A refined ultrasonic method. Appl. Opt. 1975, 14, 2099-2103. [CrossRef] [PubMed]

11. Barshan, B. Fast processing techniques for accurate ultrasonic range measurements. Meas. Sci. Technol. 2000, 11, 45-50. [CrossRef]

12. Xia, N.; Zhao, P.; Zhang, J.; Xie, J.; Fu, J. Investigation of ultrasound velocity measurements of polymeric parts with different surface roughness. Polym. Test. 2020, 81, 106231. [CrossRef]

13. Zhao, P.; Peng, Y.; Yang, W. Crystallization measurements via ultrasonic velocity: Study of poly (lactic acid) parts. J. Polym. Sci. Part B Polym. Phys. 2015, 53, 700-708. [CrossRef]

14. Jenot, F.; Ouaftouh, M.; Duquennoy, M.; Ourak, M. Corrosion thickness gauging in plates using Lamb wave group velocity measurements. Meas. Sci. Technol. 2001, 12, 1287-1293. [CrossRef]

15. Kim, Y.H.; Song, S.J.; Lee, J.K. Technique for measurements of elastic wave velocities and thickness of solid plate from access on only one side. Jpn. J. Appl. Phys. 2005, 44, 5240-5243. [CrossRef]

16. Hu, E.; Wang, W. The elastic constants measurement of metal alloy by using ultrasonic nondestructive method at different temperature. Math. Probl. Eng. 2016, 2016, 6762076. [CrossRef]

17. Zhao, P.; Xia, N.; Zhang, J. Measurement of molecular orientation using longitudinal ultrasound and its first application in in-situ characterization. Polymer 2020, 187, 122092. [CrossRef]

18. Edwards, M.J.; Bullock, E.H.; Morton, D.E. Improved precision of absolute thermal expansion measurements for ULE glass. In Proceedings of the SPIE-The International Society for Optical Engineering, Denver, CO, USA, 11 November 1996; Volume 2857, pp. 58-63.

19. Leydier, A.; Mathieu, J.; Despaux, G. The two coupling fluids method for ultrasonic velocity measurement. Application to biological tissues. Meas. Sci. Technol. 2009, 20, 095801. [CrossRef]

20. Souri, D. Ultrasonic velocities, elastic modulus and hardness of ternary $\mathrm{Sb}_{-} \mathrm{V}_{2} \mathrm{O}_{5}-\mathrm{TeO}_{2}$ glasses. J. Non-Cryst. Solids 2017, 470, 112-121. [CrossRef] 
21. Minh, H.N.; Du, J.; Raum, K. Estimation of thickness and speed of sound in cortical bone using multifocus pulse-echo ultrasound. IEEE Trans. Ultrason. Ferroelectr. Freq. Control 2019, 67, 568-579. [CrossRef] [PubMed]

22. Daugschies, M.; Rohde, K.; Glüer, C.C.; Barkmann, R. The preliminary evaluation of a $1 \mathrm{MHz}$ ultrasound probe for measuring the elastic anisotropy of human cortical bone. Ultrasonics 2014, 54, 4-10. [CrossRef] [PubMed]

23. Gulati, S.T. Mechanical properties of $\mathrm{SiO}_{2}$ vs. $\mathrm{SiO}_{2}-\mathrm{TiO}_{2}$ bulk glasses and fibers. MRS Online Proc. Libr. 1991, $244,67-84$. [CrossRef]

24. Jiang, Z. Effect of temperature on ultrasonic velocity and stress measurement (in Chinese). Nondestruct. Test. 1999, 21, 245-248.

25. Perepechko, I.; Leib, G. Acoustic Methods of Investigating Polymers; Mir Publishers: Moscow, Russia, 1975.

26. Lillie, H.R.; Ritland, H.N. Fine annealing of optical glass. J. Am. Ceram. Soc. 1954, 37, 466-473. [CrossRef] 